\title{
On the Point Spectrum of the Schrödinger Operators of Multiparticle Systems
}

\section{M. Sigal}

Department of Physics and Astronomy, Tel-Aviv University, Ramat-Aviv, Tel-Aviv, Israel

\begin{abstract}
It is shown that the point spectrum of the n-particle Schrödinger operators in the center-of-mass frame is finite for shortrange and dilation analytic potentials.
\end{abstract}

1. Let $C$ be a subset of $(1 \ldots n)$ and $H^{C}$, the Schrödinger operator of the system $C$ in its center-of-mass frame. We omit the index $C$ for $C=(1 \ldots n)$. The general structure of the spectrum of $H$ for, say, the Kato potentials is given by the following statement $^{1}$.

Theorem 1 ([1-3]). (i) The essential spectrum of the operator $H$ coincides with the half-line $[\mu, \infty)$ where ${ }^{2}$

$$
\mu=\min \left\{\lambda \mid \lambda \in \bigcup_{\left\{C_{i}\right\}} \sum_{\substack{C_{i} \subsetneq(1 \ldots n) \\ \cup C_{i}=(1 \ldots n)}} \sigma_{P}\left(\mathrm{H}^{C_{i}}\right)\right\} .
$$

(ii) The set of accumulation points of $\sigma_{P}(H)$ is included in

$$
\left\{\lambda \mid \lambda \in \bigcup_{\left\{C_{i}\right\}} \sum_{\substack{C_{i} \subsetneq(1 \ldots n) \\ \cup C_{i}=(1 \ldots n)}} \sigma_{P}\left(H^{C_{i}}\right)\right\} .
$$

The second statement was proved in the additional assumption of the dilation analyticity of the potentials (see p. 157).

2. Further and exhaustive information on the continuous spectrum of the Schrödinger operator of a multiparticle system has been obtained in scattering theory [4-7].

The next basic problem in a qualitative description of the point spectrum of the Schrödinger operator is to find classes of potentials for which the point

In this work we use the following standard definitions: the point spectrum $\sigma_{p}(A)$ of an operator $A$ is the set of all eigenvalues of finite multiplicities of $A$. The discrete spectrum $\sigma_{d}(A)$ of $A$ is the set of all isolated eigenvalues of finite multiplicities.

2 For $C=(k)$ we set $H^{C}=0$ and $\sigma_{p}\left(H^{C}\right)=\sigma\left(H^{C}\right)=\{0\}$. The union is taken over $\left\{C_{i}\right\}$ such that $\sigma_{p}\left(H^{C_{1}}\right) \neq$ $\emptyset \forall i$. 
spectrum is finite. A solution of this problem is also important for scattering theory (see $[4,5,7]$ ).

3. Familiar examples in physics indicate that one such class is apparently that of the so-called short-range potentials, i.e., potentials which decrease sufficiently rapidly at infinity. The finiteness of the discrete spectrum of $H$ for this class was proved in [8-12].

In this paper we prove the finiteness of the point spectrum of $H$ for the shortrange and dilation analytic potentials [the Conditions (i)-(iii)]. In [12] it is shown that for the isolated part of the point spectrum these assumptions may be reduced to (i).

4. In order to obtain this result we combine the method of [12] with the method of rotation of an essential spectrum of [3]. The last method separates the essential and point spectra and reduces the problem of the description of the point spectrum to the problem of the description of the discrete spectrum (but not of the self-adjoint operator). After this the method of [12] may be applied to the problem.

5. We now describe, briefly, the method of [12]. Let $A$ be an operator, the discrete spectrum of which one is interested in. Suppose that the essential spectrum of $A$ lies on some line, say, on the real axis $\mathbb{R}$. We correspond to $A$ the family

$$
A(g)=A+g W
$$

where $W$ is the compact operator such that $A(g), g \in \mathbb{R}$, has an infinite discrete spectrum if $A$ has an infinite discrete spectrum. We show that $W$ may be chosen in such a way that the accumulation points of $\sigma_{d}(A(g))$ move under changing $g$. In order to do this we find the uniformly continuous (in $\lambda$ ) family of compact operators $L(\lambda, g)$, in a Banach space, such that eigenvalues of $A(g)$ are the singular points ${ }^{3}$ of $L(\lambda, g)$ corresponding to a number $v$. Since the set of singular points of a uniformly continuous family of compact operators is closed (see, for example [12]), the accumulation points $\left\{\lambda_{i}\right\}$ of $\sigma_{d}(A(g))$ are the singular points of $L(\lambda, g)$ too for any $g$ (corresponding to the same number $v$ ). Now it is not difficult to show by the standard methods of the perturbation theory that the eigenvalue $v$ of $L\left(\lambda_{i}, g\right)$ moves continuously when $g$ changes. Hence the accumulation points of $\sigma_{d}(A(g))$ are not constant for $g \in \mathbb{R}$. On the other hand the accumulation points of $\sigma_{d}(A(g))$ belong to the essential spectrum and therefore can be situated only on its boundary. Since $\sigma_{e s}(A(g))=\sigma_{e s}(A)$ for all $g \in \mathbb{R}$ they do not depend on $g$. We obtained the contradiction to the previous statement. Hence $\sigma_{d}(A)$ has no accumulation points and therefore is finite.

6. The Schrödinger operator of an $n$-particle system in the coordinate representation, in atomic units $\left(m_{e l}=1, e=1, h=1\right)$, has the form

$$
\begin{aligned}
H_{n} f & =H_{0}^{(n)} f+V^{(n)} f \\
& =\sum_{i=1}^{n}-\frac{1}{2 m_{i}} \Delta_{i} f(x)+\frac{1}{2} \sum_{i \neq j} V_{i j}\left(x_{i}-x_{j}\right) f(x),
\end{aligned}
$$

\footnotetext{
3 The singular point of a family of compact operators $K(\lambda)$ corresponding to a number $v$ is called the value of $\lambda$ for which $v \in \sigma(K(\lambda))$
} 
where $m_{i}>0, \Delta_{i}$ is the Laplacian with respect to $x_{i}$, and the functions $V_{i j}(x)$ (in this paper) satisfy the conditions

(i) $\int \mid v_{i j}\left(\left.k\right|^{m_{0}}(1+|k|)^{\eta_{0} m_{0}} d k<C, m_{0}>3, \eta_{0}>\frac{3}{2}\left(1-2 / m_{0}\right)\right.$, where $v_{i j}(k)=\int V_{i j}(x) e^{i k x} d x$.

(ii) $V_{i j} \forall i, j$ are dilation analytic with an open connected domain $O$ of the complex plane, i.e. the operators

$$
V_{i j}(\Theta) f=V_{i j}\left(e^{\Theta}\left(x_{i}-x_{j}\right)\right) f(x), \Theta \in \mathbb{R},
$$

have an $H_{0}$-compact ${ }^{4}$ analytic continuation in $O$.

In [3] it is shown that the analyticity domain $O$ can always be extended to a complex strip

$$
O_{a}=\{z \in \mathbb{C},-a<\operatorname{Im} z<a\} .
$$

Thus we can assume that $V_{i j}(\Theta)$ are $H_{0}$-compact operator valued functions defined on a strip $O=O_{a}$. They satisfy the equality

$$
V_{i j}^{*}(\theta)=V_{i j}(\bar{\theta}) \text {. }
$$

(iii) $V_{i j}(\theta) \forall i, j$ satisfy (i) for all fixed $\Theta \in 0$.

The potentials satisfied (ii) are described in $[3,13]$. When condition (i) is satisfied, the operator $H_{n}$ is defined on $S\left(\mathbb{R}^{3 n}\right)$ and the subordination inequality holds ([14]):

$$
\left\|V^{(n)} f\right\| \leqq \varrho^{\alpha}\left\|H_{0}^{(n)} f\right\|+\varrho^{-3 n / 2}\|f\|, \alpha>0,
$$

where $\varrho>0$ is arbitrary. It follows from (3) (see [14]) that $H_{n}$ has a unique selfadjoint extension.

7. We now define certain subspaces of the configuration space $\mathbb{R}^{3 n}$ (see [15]): the subspace $R^{(0)}$ of relative movement of the system

$$
R^{(0)}=\left\{x: x=\left(x_{1} \ldots x_{n}\right) \in \mathbb{R}^{3 n}, m_{1} x_{1}+\ldots+m_{n} x_{n}=0\right\}
$$

and the subspace $R^{(c)}$ of center-of-mass movement of the system:

$$
R^{(c)}=\left\{x: x=\left(x_{1} \ldots x_{n}\right) \in \mathbb{R}^{3 n}, x_{1}=\ldots=x_{n}\right\} .
$$

Defining a scalar product in $\mathbb{R}^{3 n}$ by

$$
(x, \tilde{x})_{1}=\sum_{i=1}^{n} m_{i}\left(x_{i}, \tilde{x}_{i}\right),
$$

where $\left(x_{i}, \tilde{x}_{i}\right)$ is the usual scalar product in $\mathbb{R}^{3}$, one readily shows ([15]) that the spaces $R^{(0)}$ and $R^{(c)}$ are mutually orthogonal in the sense of this scalar product, and moreover that

$$
R^{(0)} \oplus R^{(c)}=R^{3 n} .
$$

It follows from (5) that

$$
L_{2}\left(\mathbb{R}^{3 n}\right)=L_{2}\left(R^{(0)}\right) \otimes L_{2}\left(R^{(c)}\right)
$$

4 An operator $B$ in a Hilbert space $H$ is $A$-compact if $B$ is a compact operator from $\mathscr{D}(A)$ to $H$. 
(see, e.g., [18]). This decomposition of $L_{2}\left(\mathbb{R}^{3 n}\right)$ induces a representation of the operator $H_{n}$ :

$$
H_{n}=H \otimes E^{(c)}+E^{(0)} \otimes T^{(c)},
$$

where $E^{(0)}$ and $E^{(c)}$ are the identity operators in $L_{2}\left(R^{(0)}\right)$ and $L_{2}\left(R^{(c)}\right)$, respectively. The operator $H$ is defined in $L_{2}\left(R^{(0)}\right)$ by

$$
H f=-\frac{1}{2} \Delta f+\frac{1}{2} \sum_{i \neq j} V_{i j}\left(x_{i}-x_{j}\right) f(x)=H_{0} f+V f,
$$

where $\Delta$ is the Laplacian in $L_{2}\left(R^{(0)}\right)$ [in the sense of the scalar product (4)]; the operator $T^{(c)}$ is defined in $L_{2}\left(R^{(c)}\right)$ by

$$
T^{(c)}=-\frac{1}{2} \Delta^{(c)},
$$

where $\Delta^{(c)}$ is the Laplacian in $L_{2}\left(R^{(c)}\right)$ in the sense of the scalar product (4). The subordination inequality (3) for $H_{n}$ implies (see [15]) a subordination inequality for the operator $H$ :

$$
\|V f\| \leqq \varrho^{\alpha}\left\|H_{0} f\right\|+\varrho^{-3 n / 2}\|f\|, \alpha>0,
$$

where $\varrho$ is an arbitrary positive number. By virtue of this inequality, $H$ has a unique selfadjoint extension ([17]), for which we retain the same notation $H$.

8. We now proceed to describe the compound systems, that is to say the systems derived from the original system by neglecting the interaction between certain of its subsystems.

A partition of the set $\{1, \ldots, n\}$ is defined as a collection of disjoint nonempty subsets $C_{i}$ such that $\bigcup C_{i}=\{1, \ldots, n\}$, and the term "subsystem" (cluster) for the subset $C_{i}$. Partitions will be denoted by lower case roman letters: $a, b, c, \ldots$. The number of subsets in a partition $a$ is denoted by $k(a)$, and the set of all partitions by $\mathscr{A}$; in addition, we set $\mathscr{A}_{s}=\{a \in \mathscr{A}, k(a)=s\}$. We partially order the partitions as follows. If $b$ is obtained from $a$ by breaking up certain subsystems in $a$, we write $b \subset a$ (" $b$ is contained in $a$ "). The smallest partition containing $a$ and $b$ is denoted by $a \cup b: a \cup b=\sup (a, b)$ and the largest partition contained in $a$ and by by $a \cap b: a \cap b=\inf (a, b)$.

We recall that $H^{C_{2}}$ is the Schrödinger operator of the cluster $C_{i}$ in its centerof-mass frame. For $C_{i}=(k)$ we set $H^{C_{i}}=0$ and $\sigma_{P}\left(H^{C_{i}}\right)=\sigma\left(H^{C_{2}}\right)=\{0\}$. Let

$$
\overline{\mathscr{A}}=\left\{a \in \mathscr{A}, \sigma_{P}\left(H^{C_{i}}\right) \neq \emptyset \forall C_{i} \in a\right\} .
$$

9. Now we describe a few points of the Aguilar-Balslev-Combes theory, which we use further (for details see [3]). Let $V(\Theta), \Theta \in \mathbb{R}$, be the group of operators in $L_{2}\left(R^{(0)}\right)$ defined by

$$
(V(\Theta) f)(x)=e^{\frac{1}{2} \cdot 3(n-1) \Theta} f\left(e^{\Theta} x\right) .
$$

Consider the family of operators $H(\Theta)$ defined for $\Theta \in \mathbb{R}$ by

$$
H(\Theta)=V(\Theta) H V(\Theta)^{-1}=e^{-2 \Theta} H_{0}+V(\Theta) .
$$


Since $V(\Theta)$ is $H_{0}-\varepsilon$-bounded ${ }^{5}$, this family has an analytic extension to $O$ defined by

$$
H(\Theta)=e^{-2 \Theta} H_{0}+V(\Theta), \Theta \in O .
$$

(2) implies

$$
H^{*}(\Theta)=H(\bar{\Theta}) \text {. }
$$

For $C \cong(1 \ldots n)$ we denote by $H^{C}(\Theta)$ the operator defined as $H(\Theta)$ for the system $C$.

10. The spectrum of $H(\Theta), \Theta \in O$, is described by the following

Theorem $2([3])$

(i) $\sigma_{e s}(H(\Theta))=\bigcup_{a \in \overline{\mathscr{A}}} \sum_{C_{i} \in a} \sigma_{d}\left(H^{C_{i}}(\Theta)\right)+e^{-2 i \operatorname{Im} \Theta} \mathbb{R}^{+}$.

(ii) $\sigma_{d}(H(\Theta)) \cap \mathbb{R}$ is constant for $\Theta \in O$ and equal to $\sigma_{p}(H) \backslash \bigcup_{a \in \bar{A}} \sum_{C_{l} \in a} \sigma_{p}\left(H^{C_{\imath}}\right)$.

(iii) The set of accumulation points of $\sigma_{d}(H(\Theta)) \cap \mathbb{R}$ is included in $\bigcup_{a \in \overline{\mathscr{A}}} \sum_{C_{i} \in a} \sigma_{d}\left(H^{C_{i}}(\Theta)\right) \cap \mathbb{R}=(b y(\mathrm{ii}))=\bigcup_{a \in \overline{\mathscr{A}}} \sum_{C_{\mathrm{r}} \in a} \sigma_{p}\left(H^{C_{i}}\right)$.

Further we assume that $\Theta \in O \backslash \mathbb{R}$ and is fixed.

11. We associate with $H$ a two-parameter family of Berezin's operators $L(z, \Theta)=L_{H}(z, \Theta)([16]$, see also [17]), defined by induction on partitions $a$ in the space $\mathscr{D}\left(H_{0}\right)$ :

$$
\begin{aligned}
& L_{u}(z, \Theta)=e^{2 \Theta} R_{0}\left(z e^{2 \Theta}\right) V_{\alpha}(\Theta), u=u_{\alpha}=\left\{(\alpha) \prod_{k \notin \alpha}(k)\right\} \in \mathscr{A}_{n-1}, \\
& E+L_{a}(z, \Theta)=\prod_{s=k(a)+1} \prod_{b \in \mathscr{A}_{s}, b \subset a}\left(E+L_{b}(z, \theta)\right)\left(E+\sum_{b \in \mathscr{A}_{n-1}, b \subset a} L_{b}(z, \theta)\right), \\
& 1 \leqq k(a)<n-1
\end{aligned}
$$

$$
L(z, \Theta)=L_{a}(z, \Theta)\left(a \in \mathscr{A}_{1}\right) .
$$

The symbol $\prod_{b \in \mathscr{A}_{s}}$ denotes a product of the appropriate operator factors in some arbitrary but fixed order. It is not difficult to see by induction that if $z$ lies outside the set $\sum_{C_{i} \in a} \sigma_{e s}\left(H^{C_{i}}(\Theta)\right)$, then $L_{a}(z, \Theta)$, is a bounded operator in $\mathscr{D}\left(H_{0}\right)$ and $E+$ $L_{a}(z, \Theta), z \notin \sum_{C_{\imath} \in a} \sigma\left(H^{C_{\imath}}(\Theta)\right)$, has a bounded inverse. We set

$$
F(z, \Theta)=\prod_{s=1}^{n-1} \prod_{a \in \mathscr{A}_{s}}\left(E+L_{a}(z, \Theta)\right)^{-1} e^{2 \Theta} R_{0}\left(z e^{2 \Theta}\right) .
$$

It is evident from (8) and (9) that the operators $L(z, \Theta)$ and $H(\Theta)-z E$ are related by

$$
E+L(z, \Theta)=F(z, \Theta)(H(\Theta)-z E) \text {. }
$$

5 An operator $B$ is $A-\varepsilon$-bounded if the following inequality is valid

$\|B f\| \leqq \varepsilon\|A f\|+b(\varepsilon)\|f\|, f \in \mathscr{D}(A)$,

$A$ compactness implies $A-\varepsilon$-boundness. 
12. If $z$ lies in the continuous spectrum of $H(\Theta)$, the operator $L(z, \Theta)$ is no longer bounded on $\mathscr{D}\left(H_{0}\right)$. We shall be interested in the limit of the family of operators $L(\lambda, \Theta)$ as $\lambda$ approaches $\sigma_{e s}(H(\Theta)) \cap \mathbb{R}$. This limit will be studied in specially chosen scales of Banach spaces $B_{\Theta}^{(a)}, B, a \in \bar{A}$, whose construction is given in the Appendix.

Throughout this work we shall assume that Condition A formulated in the Appendix is fulfilled by the potentials ${ }^{6}$.

$$
\begin{aligned}
& \sum_{C_{i} \in a}^{\text {Let }} \sigma_{p}\left(H^{C_{i}}\right)=\left\{\lambda^{a}\right\}, a \in \overline{\mathcal{A}}, \text { and } \\
& x^{a}=(3 / 4) \min \left\{\left|\lambda^{a}-\lambda^{b}\right|, \lambda^{b} \neq \lambda^{a}, \lambda^{b} \in \sum_{C_{i} \in b} \sigma_{p}\left(H^{C_{i}}\right)\right\} .
\end{aligned}
$$

13. Lemma 1. The operator $L(\lambda, \Theta),\left|\lambda-\lambda^{a}\right| \leqq x^{a}$, is uniformly bounded, continuous in the operator topology with respect to $\lambda$, and compact in $B_{\Theta}^{(a)}, a \in \overline{\mathscr{A}}$.

Lemma 2. The operator $F(\lambda, \Theta),\left|\lambda-\lambda^{a}\right| \leqq \chi^{a}$, is bounded from $B$ to $B_{\Theta}^{(a)}$; for $\lambda=\lambda^{a}$ the operator has an inverse which maps $\bar{B}_{\Theta}^{(a)}$ continuously into $B, a \in \bar{A}$.

Lemma 3. The operator $H(\Theta)-\lambda^{a} E$ is continuous from $B_{\Theta}^{(a)}$ to $B, a \in \overline{\mathscr{A}}$.

The proofs of these lemmas are exactly the same as those of Lemmas 1.1-1.3 of [12].

14. Let $\mathscr{W}$ denote the class of operators in $L_{2}\left(R^{(0)}\right)$ satisfying the conditions: (a) the operators of $\mathscr{W}$ are finite-dimensional, (b) the operators of $\mathscr{W}$ are continuous from $L_{2}\left(R^{(0)}\right)$ to $S\left(R^{(0)}\right)$, (c) $W(\Theta)=V(\Theta) W V(\Theta)^{-1}, \Theta \in \mathbb{R}, W \in \mathscr{W}$, have an analytical extension to $O$, as continuous operators from $L_{2}\left(R^{(0)}\right)$ to $S\left(R^{(0)}\right)$.

Consider the family of operators

$$
H(g, \Theta)=H(\Theta)+g W(\Theta), W \in \mathscr{W}, g \in \mathbb{R} .
$$

With the family $H(g, \Theta)$ we associate a three-parameter family of operators $L(z, g, \Theta)$ defined by

$$
L(z, g, \Theta)=L(z, \Theta)+g F(z, \Theta) W(\Theta)
$$

It follows from (10), (12) that

$$
E+L(z, g, \Theta)=F(z, \Theta)(H(g, \Theta)-z E)
$$

15. Using Eq. (13) and obvious estimates for eigenfunctions of the discrete spectrum of $H(g, \Theta)$ one obtains the following:

Lemma 4. If $\lambda(g)$ is a point of the real discrete spectrum of $H(g, \Theta)$, then the operator $L(\lambda(g), g, \Theta)$ has the eigenvalue -1 .

16. Lemma 5. Assume that the operator $L\left(\lambda^{a}, \Theta\right)$ has an eigenvalue -1 . Then there exists a positive number $\varepsilon$ and a subset $\tilde{\mathscr{W}}$ of $\mathscr{W}$ such that $-1 \notin \sigma\left(L\left(\lambda^{a}, g, \Theta\right)\right)$ for $g \in(0, \varepsilon)$ and $W \in \tilde{\mathscr{W}}$.

Proof. We evaluate the first derivatives with respect to $g$, at the point $g=0$, of the eigenvalues of $L\left(\lambda^{a}, g, \Theta\right)$, which equal -1 at $g=0$.

6 See note added in proof. 
Let $\mathscr{L}_{\Theta}^{(a)}$ be the eigensubspace of the operator $L\left(\lambda^{a}, O, \Theta\right)=L\left(\lambda^{a}, \Theta\right)$ that corresponds to the point -1 of the spectrum, and $\psi(\Theta)$ an arbitrary function in $\mathscr{L}_{\Theta}^{(a)}$ for which there exists an eigenfunction $\psi(g, \Theta)$ of $L\left(\lambda^{a}, g, \Theta\right)$ such that $\psi(O, \Theta)=\psi(\Theta)$. Let $\lambda_{\psi}(g)$ be the eigenvalue corresponding to $\psi(g, \Theta), \lambda_{\psi}(0)=-1^{7}$.

The following equation is obtained using (7) and Lemmas 1-3 exactly in the same way as the corresponding equation in $[6]^{8}$ :

$$
\left.\left(d \lambda_{\psi}(g) / d g\right)\right|_{g=0}=(W(\Theta) \psi(\Theta), \psi(\bar{\Theta})) /\left(F^{-1}\left(\lambda^{a}, \Theta\right) \psi(\Theta), \psi(\bar{\Theta})\right) .
$$

We assume that, in addition to (a)-(c), the operator $W(\Theta)$ satisfies the condition: $W(\Theta)$ is strictly negative on $\mathscr{L}_{\Theta}^{(a)}$, i.e., there exists $C>0$ such that

$$
(W(\Theta) \psi(\Theta), \psi(\bar{\Theta}))<-C, \psi(\Theta) \in \mathscr{L}_{\Theta}^{(a)} .
$$

But by Lemma 2

$$
\left|\left(F^{-1}\left(\lambda^{a}, \Theta\right) \psi(\Theta), \psi(\bar{\Theta})\right)\right|<\infty, \psi(\Theta) \in B_{\Theta}^{(a)},
$$

and so it follows from (14) and (15) that for any function $\psi(\Theta) \in \mathscr{L}_{\Theta}^{(a)}$

$$
\left.\left(d \lambda_{\psi}(g) / d g\right)\right|_{g=0} \neq 0 .
$$

The last implies the statement of the lemma.

17. Having prepared all the necessary material for the proof of our main theorem, we now proceed to state and prove it.

Theorem 3. Let the potentials $V_{i j}$ in the operator $H$ satisfy (i)-(iii) and Condition $A$. Then the point spectrum of $H$ is finite.

Proof. Suppose, on the contrary, that $H$ has an infinite point spectrum. By Theorem 2 the point spectrum of $H$ outside the set

$$
\bigcup_{a \in \mathscr{A}} \sum_{C_{i} \in a} \sigma_{p}\left(H^{C_{i}}\right)
$$

coincides with the discrete spectrum of $H(\Theta), \Theta \in O \backslash \mathbb{R}$, the accumulation points of which is contained in (16).

Define $H(g, \Theta), L(z, g), \mathscr{L}_{\Theta}^{(a)}$ and $\lambda_{\psi}(g), \psi(\Theta) \in \mathscr{L}_{\Theta}^{(a)}$, as before

Since $W(\Theta)$ is a compact operator in $L_{2}\left(R^{(0)}\right)$, it follows that the operator $H(g, \Theta)$ has the same limit spectrum as $H(\Theta)$ for any finite $g$. Since $W$ is a finitedimensional operator, it thus follows that for any $g$ the operator $H(g, \Theta)$ has an infinite discrete spectrum, whose accumulation points are precisely the same as that of $\sigma_{p}(H)$. Let $\lambda_{n}(g), n=1,2, \ldots$, denote the eigenvalues of $H(g, \Theta)$ belonging to the discrete spectrum and accumulating to the point $\lambda^{a}, a \in \overline{\mathscr{A}}$ :

$$
\lambda_{n}(g) \rightarrow \lambda^{a} \quad \text { as } n \rightarrow \infty, g \in \mathbb{R} .
$$

It follows from Lemma 4 that

$$
\sigma\left(L\left(\lambda_{n}(g), g, \Theta\right)\right) \ni-1, g \in \mathbb{R} .
$$

It is not difficult to show that $\lambda_{t p}(g)$ does not depend on $\Theta$; therefore we omit index $\Theta$ in notation. $(W(\Theta) \psi(\Theta), \psi(\bar{\Theta}))$ and $\left(F^{-1}\left(\lambda^{a}, \Theta\right) \psi(\Theta), \psi(\bar{\Theta})\right)$, in fact, do not depend on $\Theta$. 
By (17), (18), Lemma 1 and the theorem stating that the set of singular points of a compact operator is closed (see Appendix to [12]), it follows that

$$
\sigma\left(L\left(\lambda^{a}, g, \Theta\right)\right) \ni-1, g \in \mathbb{R},
$$

for any operator $W$ in $\mathscr{W}$. But this contradicts Lemma 5 . Hence the point spectrum of $H$ outside of the set (16) is finite. Hence the entire point spectrum of $H$ if finite. Theorem 3 is proved.

\section{Appendix}

1. In this appendix we define scales of Banach spaces in which the operators $L(z, \Theta), F(z, \Theta)$ are studied. We describe these scales in the so-called momentum representation. This representation is obtained from the more familiar coordinate representation by the Fourier transformation $F$ of the basic space $L_{2}\left(\mathbb{R}^{3 n}\right)$ and the corresponding transformation $F H_{n} F^{-1}$ of the operator $H_{n}$. We shall therefore translate some of our definitions into the language of the momentum representation and add some new definitions. The old notation will be retained for the operators $H^{C}$ in the new representation; this will involve no confusion since all our deliberations will be in the momentum language.

2. We define a scalar product in the momentum space $\mathbb{R}^{3 n}$ (see [7]) which is dual (in the sense of the Fourier transform) to the scalar product (4) in the configuration (coordinate) space:

$$
(p, \tilde{p})^{\prime}=\sum_{i=1}^{n} m_{i}^{-1}\left(p_{i}, \tilde{p}_{i}\right)
$$

To each partition $a$ there corresponds a pair of spaces $R^{a}$ and $R_{a}$ (the space of relative movement of particles in subsystems of $a$ and the space of the $C M$ movement of these subsystems), defined by

$$
\begin{aligned}
R^{a} & =\left\{p: p=\left(p_{1} \ldots p_{n}\right) \in \mathbb{R}^{3 n}, \sum_{k \in C_{\imath}} p_{k}=0 \forall C_{i} \in a\right\}, \\
R & =R^{a} \quad(k(a)=1)
\end{aligned}
$$

and

$R_{a}=\left\{p: p=\left(p_{1} \ldots p_{n}\right) \in R, m_{i}^{-1} p_{i}=m_{j}^{-1} p_{j}\right.$ if there exists $C_{k} \in a$ such that $\left.i, j \in C_{k}\right\}$.

With these definitions we have (in the sense of the scalar product (A1))

$$
R^{a} \perp R_{a}, R^{a} \oplus R_{a}=R .
$$

If $k(a)=1$, the spaces $R^{a}$ and $R_{a}$ are the Fourier-duals of $R^{(0)}$ and $R^{(c)}$, respectively. It follows from (A2) that

$$
L_{2}(R)=L_{2}\left(R^{a}\right) \otimes L_{2}\left(R_{a}\right) .
$$

We define

$$
J_{a}=\left\{b \in \overline{\mathscr{A}}, \sum_{C_{i} \in b} \sigma_{p}\left(H^{C_{i}}\right) \ni \lambda^{a}\right\} \cup \mathscr{A}_{n}
$$


and

$$
\left(\mathscr{N}_{a}(\eta) f\right)\left(p_{a}\right)=\mathscr{N}_{a}\left(p_{a} ; \eta\right) f\left(p_{a}\right), a \in \mathscr{A} \backslash \mathscr{A}_{1},
$$

where $\mathcal{N}_{a}\left(p_{a} ; \eta\right)$ are the estimating functions introduced in [17]. We recall one property of these functions:

$$
\int\left[\mathscr{N}_{a}\left(p_{a} ; \eta\right)\right]^{k} d p_{a}<C, \eta k>3 .
$$

The Banach spaces in question are defined as follows:

$$
B_{m, \eta}\left(R_{a}\right)=\mathscr{N}_{a}(\eta) L_{m}\left(R_{a}\right),\|f\|_{B_{m, \eta}\left(R_{a}\right)}=\left\|\mathscr{N}_{a}^{-1}(\eta) f\right\|_{L_{m}\left(R_{a}\right)},
$$

and

$$
\hat{B}_{m, \eta}^{(a)}=\sum_{b \in J_{a}} \oplus B_{m, \eta}\left(R_{b}\right),\|\hat{f}\|_{\hat{B}_{m, \eta}^{(a)}}=\sum_{b \in J_{a}}\left\|f_{b}\right\|_{B_{m, \eta}\left(R_{b}\right)}, \hat{f}=\left\{f_{b}, b \in J_{a}\right\}
$$

We define a linear mapping of $\hat{B}_{m, \eta}^{(a)}$ by

$$
\Pi^{(a)}(\Theta) \hat{f}=\sum_{b \in J_{a}} \psi^{b}\left(p^{b} ; \theta\right) f_{b}\left(p_{b}\right) /\left(\tau\left(p_{b}\right)-\lambda^{a} \delta_{k(b), n}\right),
$$

where we used the notation

$$
\tau(p)=1 / 2(p, p)^{\prime}, p \in R .
$$

Denote the image of $\hat{B}_{m, \eta}^{(a)}$ under the homomorphism $\Pi^{(b)}(\Theta)$ by

$$
B_{m, \eta, \Theta}^{(a)}=\Pi^{(a)}(\Theta) \hat{B}_{m, \eta}^{(a)} .
$$

$B_{m, \eta, \Theta}^{(a)}$ becomes a Banach space if we define the norm by

$$
\|f\|_{B_{m, \eta}^{(a), \Theta}}=\inf _{\Pi^{(a)}(\Theta) \hat{f}=f}\|\hat{f}\|_{\hat{B}_{m, \eta}^{(m)},} .
$$

4. $B_{m, \eta, \Theta}^{(a)}$ and $B_{m, \eta}(R)$ are the desired scales of Banach spaces. The following inbeddings are valid for them

$$
B_{m^{\prime}, \eta^{\prime}, \Theta}^{(a)} \subset B_{m, \eta, \Theta}^{(a)}, B_{m^{\prime}, \eta^{\prime}}(R) \subset B_{m, \eta}(R), m^{\prime} \geqq m, \eta^{\prime}>\eta+3\left(1 / m-1 / m^{\prime}\right) .
$$

When $m^{\prime}>m$, these imbeddings are compact. The spaces $B_{\Theta}^{(a)}$ and $B$ of the main text are $B_{m, \eta, \Theta}^{(a)}$ and $B_{m, \eta}(R)$, respectively, for any fixed induces $m, \eta$, subordinated by the conditions

$$
m>6, \eta>3 / 2(1-2 / m) \text {. }
$$

5. Now, in conclusion, we formulate the additional restriction on the operator $H$. Let

$$
\mathscr{A}=\left\{a \in \mathscr{A}, k(a)>1, \exists b \subsetneq a: \sigma_{p}\left(H^{C_{i}}\right) \neq \emptyset \forall C_{i} \in b\right\} .
$$

Let $L^{a}(z, \Theta)$ denote the restriction of the operator $L_{a}(z, \Theta)$ to $\mathscr{D}\left(H^{a}\right)$. 
Condition $A$. The potentials $V_{i j}(x)$ have the property that the equation $\varphi+$ $L^{a}\left(\lambda^{c}, \theta\right) \varphi=0$ has no nontrivial solutions of the form ${ }^{9}$

$$
\sum_{\substack{b \subset a \\ b \in J_{c}}} \psi^{b}\left(p^{b}, \Theta\right) f_{b}\left(p_{b}^{a}\right) /\left(\tau\left(p_{b}^{a}\right)-\lambda^{c} \delta_{k(b), n}\right), f_{b} \in \mathcal{N}_{b}^{a}(\eta) L_{m}\left(R_{b} \cap R^{a}\right), 6<m<2 m_{0},
$$

$\eta>3 / 2(1-2 / m)$, for any $a$ in $\mathscr{A}$ and $c \subset a, c \in \overline{\mathscr{A}}, J_{c} \cap \mathscr{A}_{k(a)+1} \neq \emptyset$.

9 For the definition of the estimating functions $\mathcal{N}_{b}^{a}\left(p_{b}^{a} ; \Theta\right)$, see [17]. We note here only that

$\int\left[\mathcal{N}_{b}^{a}\left(p_{b}^{a}, \eta\right)\right]^{k} d p_{b}^{a}<C, k \eta>3$.

\section{References}

1. Zhislin, G. M.: Trudy Mosk. Mat. Obsc. 9, 81 (1960)

2. Hunziker, W.: Helv. Phys. Acta 39, 451 (1966)

3. Aguilar, J., Combes, J. M.: Commun. math. Phys. 22, 269 (1971); Balslev, E., Combes, J. M.: Commun. math. Phys. 22, 280 (1971); van Winter, C.: University of Kentucky (preprint) (1971)

4. Faddeev, L.D.: Mathematical aspects of the threebody problem in the quantum theory of scattering. Israel Program of Scientific Translations, Jerusalem, 1965

5. Hepp, K.: Helv. Phys. Acta 42, 425 (1969)

6. Lavine, R. B.: Commun. math. Phys. 20, 301 (1970)

7. Sigal,I.M.: Institute of Theor. Phys. Akademia Nauk USSR, Kiev Preprint-74-19P (1974). Tel-Aviv University (preprint) (1975)., Trans. Amer. Math. Soc. (submitted for publication)

8. Zhislin, G. M.: Theor. Math. Phys. 7, 571 (1971)

9. Zhislin,G. M.: Soviet Math. Dokl. 13, 1445 (1972)

10. Jafaev, D. R.: Funct. Anal. Appl. 6, 396 (1972)

11. Antonets, M.A., Zhislin, G. M., Shereshevskii, I. A.: Theor. Math. Phys. 16, 800 (1974)

12. Sigal, I. M. : Tel-Aviv University (preprint) (1975), submitted for publication

13. Babbit, D., Balslev, E.: Commun. math. Phys. 35, 173 (1974)

14. Kato, T.: Trans. Amer. Math. Soc. 70, 195 (1951)

15. Sigalov, A. G., Sigal, I. M.: Teor. Math. Fiz. 5, 73 (1970); Theor. Math. Phys. 5, 990 (1971)

16. Berezin, F. A.: Soviet Math. Dokl. 6, 997 (1965)

17. Sigal,I. M.: Soviet Math. Dokl. 13, 756 (1972)

18. Berezanskii, Ju. M.: Expansion in eigenfunctions of self-adjoint operators Providence. Rhode Island: Am. Math. Soc. 1965

Communicated by W. Hunziker

Received September 1, 1975

Note Added in Proof. Condition A cannot be effectively controlled, but it is not so important because for any potentials $\left\{V_{\alpha}\right\}$, satisfying (i)-(iii), there exist a number $\varepsilon>0$ and a subset $\mathfrak{U} \subset\{(i j)$, $i, j=1 \ldots n\}$ such that Condition A is satisfied for the Hamiltonian $H(g)=H_{0}+\sum V_{\alpha}+\sum_{\alpha \in \mathscr{A}} g_{\alpha} V_{\alpha} \forall g=$ $\left(g_{\alpha}, \alpha \in \mathfrak{U}\right):\left\{0<\left|g_{\alpha}\right| \leqq \varepsilon\right\}$ (I. M.Sigal, unpublished). 